\title{
Endlich Subsidiarität? Die parlamentarische Subsidiaritätskontrolle am Beispiel von „Monti II“
}

\author{
Martin Höpner / Lena Ehret
}

\begin{abstract}
Subsidiarity, finally? How the early warnings system worked in the conflict over "Monti II" Abstract: Three hopes are associated with the new parliamentary early warnings system: It is hoped that the principle of subsidiarity will gain effectiveness, that the European political system will gain legitimacy, and that the pre-constitutional preconditions of a European democracy will further develop. In order to derive insights about the validity of these hopes, this article conducts an in-depth analysis of the conflict over the Commission's "Monti II" proposal. "Monti II" was the first regulation proposal against which more than one third of member states' parliaments raised reasoned opinions (the so-called "yellow card" procedure). The analysis shows that the hopes rest upon far-reaching premises with respect to the origins of the breaches of subsidiarity and with respect to the nature of the respective conflict lines. The hopes, therefore, go much too far.
\end{abstract}

Keywords: European Union, European integration, subsidiarity, comparative political economy, right to strike

Schlagwörter: Europäische Union, europäische Integration, Subsidiarität, Vergleichende Politische Ökonomie, Streikrecht

\section{Einleitung}

Die Aufwertung nationaler Parlamente im politischen System der Europäischen Union (EU) gilt als eine der entscheidenden Innovationen des im Jahr 2009 in Kraft getretenen Lissabonner Reformvertrags. Im Rahmen der Subsidiaritätsrüge werden die mitgliedstaatlichen Parlamente zu Initiatoren eines eigenständigen Kontrollverfahrens, das einsetzt, bevor umstrittene Gesetzesvorhaben den Rat erreichen. Erstmals eröffnet sich den nationalen Parlamenten damit ein Einflusskanal, der sich ohne Umweg über die mitgliedstaatlichen Regierungen direkt an die europäischen Organe richtet. Im Ergebnis können die Parlamente der EUMitgliedstaaten Verstöße gegen das Prinzip der Subsidiarität daher nun bereits im Frühstadium und alleinverantwortlich ahnden.

Auftrag und Ermächtigung zur Subsidiaritätskontrolle gehen aus Art. 12 EUV hervor, die Details finden sich in dem Protokoll (Nr. 2) über die Anwendung der Grundsätze der Subsidiarität und der Verhältnismäßigkeit, das ebenso wie der eigentliche Vertrag Teil des geschriebenen Primärrechts ist. ${ }^{1}$ In einem Zeitraum von acht Wochen nach Übermittlung eines Richtlinien- oder Verordnungsentwurfs

Für wertvolle Hinweise danken wir Aleksandra Maatsch, Oliver Treib, Fritz Scharpf und zwei anonymen Gutachtern der Politischen Vierteljahresschrift.

1 Der Wortlaut findet sich unter http://www.flegel-g.de/lissabon/prot2-lissabon.html (Zugriff: 08.06.2016).

PVS, 57. Jg., 3/2016, S. 403-429, DOI: 10.5771/0032-3470-2016-3-403

403

\section{MPIfG Journal Article}

Martin Höpner, Lena Ehret: Endlich Subsidiarität? Die parlamentarische Subsidiaritätskontrolle am Beispiel von „Monti II“. In:

Politische Vierteljahresschrift 57(3), 403-429 (2016). Nomos

The original publication is available at the publisher's web site: http://dx.doi.org/10.5771/0032-3470-2016-3-403

The MPIfG Journal Articles series features articles by MPIfG researchers and visiting scholars published in peer-reviewed journals. Max Planck Institute for the Study of Societies (MPIfG) Cologne | www.mpifg.de 
durch die Kommission haben die nationalen Parlamente Gelegenheit, eine Stellungnahme abzugeben, in der sie darlegen, warum das Gesetzesvorhaben ihrer Ansicht nach nicht mit dem Grundsatz der Subsidiarität vereinbar ist. Hierbei hat jeder Mitgliedstaat zwei Stimmen, die im Fall bikameraler Legislativen zwischen der ersten und der zweiten Kammer aufgeteilt werden. Erreicht die Anzahl begründeter Stellungnahmen das Quorum von einem Drittel der den Parlamenten zugewiesenen Stimmen, muss die Kommission ihren Vorschlag überprüfen und, sofern sie weiterhin an diesem festhalten will, ihrerseits eine Begründung abgeben. Dieses mitunter als "gelbe Karte" bezeichnete Verfahren steht im Zentrum der vorliegenden Betrachtungen. Bisher gaben nationale Parlamente 304 begründete Stellungnahmen zu europäischen Gesetzesinitiativen ab. ${ }^{2}$ Nur dreimal wurde hierbei allerdings die Schwelle zur "gelben Karte" überschritten, und zwar erstmals im Mai 2012, als es um die so genannte „Monti II"-Verordnung ging, dann noch einmal im Oktober 2013, als 13 Parlamente aus 11 Mitgliedstaaten begründete Stellungnahmen gegen den Vorschlag für eine Verordnung zur Errichtung einer Europäischen Staatsanwaltschaft abgaben, und schließlich noch einmal im Mai 2016, als 15 Parlamente aus 12 Ländern den Kommissionsvorschlag für eine Überarbeitung der Entsenderichtlinie rügten (siehe hierzu das Nachwort zu diesem Text).

Das neue Instrument ist zudem mit zwei weiteren - bisher nicht genutzten Hebeln ausgestattet: Wird ein Quorum von 50 Prozent der Stimmen erreicht, muss die Kommission ihr etwaiges Festhalten an dem Vorschlag auch gegenüber dem Europäischem Parlament (EP) und dem Rat eigens begründen; halten Mehrheiten in EP und Rat den Grundsatz der Subsidiarität ebenfalls für verletzt, wird der Vorschlag nicht weiter behandelt („rote Karte"). ${ }^{3}$ Außerdem ist der Subsidiaritätsrüge eine Subsidiaritätsklage an die Seite gestellt: Jede stimmberechtigte nationale Kammer kann nach Abschluss eines Gesetzgebungsverfahrens vom Europäischen Gerichtshof (EuGH) überprüfen lassen, ob sich die betreffende europäische Gesetzgebung im Einklang mit dem Subsidiaritätsprinzip befindet.

Drei Hoffnungen ruhen auf dem neuen Instrument. Die naheliegendste dieser Hoffnungen ist die auf eine bessere Durchsetzung des Prinzips der Subsidiarität, das aus der katholischen Soziallehre stammt und besagt, dass Probleme in Mehrebenenstrukturen im Zweifel von der untersten Ebene - hier also: den Mitgliedstaaten - bearbeitet werden sollen (Richter 1987, S. 296-300). Nur wo es zur Problembearbeitung zwingend notwendig ist, soll die nächsthöhere Ebene handeln. Zwar wurde das Subsidiaritätsprinzip bereits im Maastrichter Vertrag als eines der leitenden Ziele der Europäischen Gemeinschaft (nachfolgend stets: der EU) benannt. Über die Einhaltung dieses Prinzips hatten aber primär die Unionsorgane selbst zu wachen, denen also im Fall von Verstößen aufgegeben wurde, gegen ihr eigenes Tätigwerden einzuschreiten. Da die Unionsorgane in Zuständig-

2 Stand: Ende August 2015. Datenquelle: http://www.epgencms.europarl.europa.eu/cmsdata/ upload/417e8af9-a828-429b-9876-4045cd6598e3/State_of_play_-_October_2015.pdf (Zugriff: 08.06.2016).

3 Anzumerken ist freilich, dass ein Richtlinien- oder Verordnungsvorschlag die betreffenden Organe in einer solchen Konstellation ohnehin nicht erfolgreich passieren könnte. Die "rote Karte" bewirkt gegenüber dem status quo ante daher keine praktische Veränderung. 
keitsfragen im Ergebnis als „Kontrolleure in eigener Sache“ fungierten, wurde der status quo ante nicht als hinreichend empfunden, um einer etwaigen schleichenden - subsidiaritätswidrigen - Zentralisierung der EU entgegenzuwirken (Shirvani 2010, S. 753). Vor diesem Hintergrund erscheint es konsequent, dass nunmehr den eigentlichen Verlierern etwaiger Subsidiaritätsverletzungen ein eigener Kontrollkanal zugewiesen wurde: den nationalen Parlamenten.

Eine zweite Hoffnung besteht in einer verbesserten Legitimationszufuhr an die europäischen Organe (siehe z. B. Boronska-Hryniewiecka 2013, S. 182). Allgemein lassen sich auf der Input-Seite ${ }^{4}$ zwei Legitimitätsquellen der europäischen Politik unterscheiden: eine, die von Wahlen zu den nationalen Parlamenten über die im Rat vertretenen Regierungen der Mitgliedstaaten verläuft, und eine weitere, die über die EP-Wahlen und die Beteiligung des EPs an der europäischen Gesetzgebung ohne Umweg über die mitgliedstaatlichen Demokratien direkt an der europäischen Ebene ansetzt. Funktioniert der neue Kontrollmechanismus, dann öffnet er einen neuen, von diesen beiden Quellen zu unterscheidenden Legitimationskanal. Die Subsidiaritätskontrolle geht von den nationalen Parlamenten aus, stärkt aber gleichwohl nicht den intergouvernementalen Arm der EU. Vielmehr treten die Parlamente als „virtuelle dritte Kammer" (Cooper 2012) neben EP und Rat, üben eine eigenständige Kontrollaufgabe aus und binden die europäische Politik im Ergebnis effektiver als vorher an den Willen der europäischen Bürgerinnen und Bürger. ${ }^{5}$ Am Ende steht, so die Hoffnung, ein Mehr an demokratischer Input-Legitimität.

Auch die dritte Hoffnung richtet sich auf die Demokratie, ist aber langfristiger Natur: Das neue Verfahren, so die Hoffnung, kann einen Beitrag zur Weiterentwicklung der vor-rechtlichen, vor-konstitutionellen Voraussetzungen demokratischer Prozesse auf europäischer Ebene leisten (Leitfeld u. Malang 2014, S. 29; Semmler 2010, S. 534-536). Bisher handelt es sich bei den europäischen Parteienfamilien um vergleichsweise lockere Zusammenschlüsse mit wenig horizontaler Koordination zwischen den Parteiführungen und schwach ausgeprägter programmatischer Kohärenz. Die Verfügbarkeit der Subsidiaritätskontrolle erhöht die auf die nationalen Parteien gerichteten Anreize, sich effektiv zu koordinieren. Ohne eine solche Koordinationsleistung erscheint es unwahrscheinlich, dass sich in ausreichend vielen nationalen Parlamenten die notwendigen Mehrheiten für die Abgabe begründeter Stellungnahmen finden. Je lebhafter und effektiver der transnationale Austausch zwischen den programmatisch verwandten nationalen Schwesterparteien, umso größer die Chance auf die Entwicklung intern zunehmend kohärenter und im Parteienvergleich zunehmend unterscheidbarer Positionen, die den Bürgerinnen und Bürgern in europäischen Wahlen zur Auswahl vorgelegt werden können.

4 Hiervon sind outputseitige Legitimationsquellen zu unterscheiden: Entscheidungen werden als legitim empfunden, wenn ihre Ergebnisse mit den Präferenzen der Betroffenen übereinstimmen (grundlegend: Scharpf 1999, S. 16-28).

5 Wie Groen und Christiansen (2015, S. 43-45) darlegen, wurde die Schaffung einer formellen, aus Mitgliedern der nationalen Parlamente zusammengesetzten neuen Kammer im Vorfeld des Lissabonner Vertrags als Alternative zur Ermächtigung der nationalen Parlamente zur Subsidiaritätsrüge diskutiert. 
Unsere nachfolgenden Betrachtungen verstehen wir als Beitrag zur Diskussion über die Berechtigung dieser Hoffnungen. Wir führen eine Tiefenbetrachtung von einem der drei Fälle durch, bei denen die mitgliedstaatlichen Parlamente bei der Abgabe ihrer begründeten Stellungnahmen das Quorum der "gelben Karte" erreichten. Dabei handelt es sich um den Konflikt über den Verordnungsvorschlag „Monti II“, der das Spannungsfeld zwischen Binnenmarktfreiheiten und nationalem Arbeitsrecht zum Gegenstand hatte. Die Tiefenanalyse des Einzelfalls öffnet die Augen dafür, dass den mit dem neuen Instrument verknüpften Hoffnungen unausgesprochene Prämissen zugrunde liegen. Legt man diese offen, dann zeigt sich, dass die Effektivität des neuen Instruments höchst voraussetzungsvoll ist und dass die Hoffnungen also eingehend zu qualifizieren sind.

Um unser Argument zu entfalten, wenden wir uns in Abschnitt 2 zunächst dem europarechtlichen und politikwissenschaftlichen Diskussionsstand zu den Potenzialen des neuen Kontrollinstruments zu. In Abschnitt 3 arbeiten wir die Genese des durch „Monti II" aktualisierten Konflikts heraus und zeigen auf, dass der von den nationalen Parlamenten als Subsidiaritätsverstoß empfundene Eingriff in die mitgliedstaatliche Autonomie nicht von der europäischen Gesetzgebung ausging, sondern von der Rechtsprechung des Europäischen Gerichtshofs (EuGH). Denn der Kommissionsvorschlag für eine "Monti II"-Verordnung zielte im Kern auf die Kodifizierung der zuvor ergangenen Rechtsprechung. Die „gelbe Karte“ der mitgliedstaatlichen Parlamente war daher von vornherein an den falschen Adressaten gerichtet und konnte den Subsidiaritätsschutz nicht effektuieren.

In Abschnitt 4 nutzen wir das Rügeverhalten der Parlamente, um genaueren Aufschluss über die Lage der den Fall strukturierenden Konfliktlinie zu erhalten. Wie sich dort zeigt, handelte es sich nicht um einen Konflikt zwischen "linken" und "rechten" Integrationsvorstellungen. Die Schärfung einer solchen Konfliktlinie könnte in der Tat einen Beitrag zur Stärkung der internen Kohärenz der europäischen Parteienfamilien leisten. Vielmehr handelte es sich um einen Konflikt zwischen Ländergruppen mit unterschiedlichen Interessen an der Regulierung der transnationalen Arbeitnehmerentsendung. Die verbesserte Artikulationsfähigkeit entlang solcher Konfliktlinien trägt zur erhofften Schärfung der programmatischen Kohärenz europäischer Parteien nichts bei. Insgesamt, so argumentieren wir im abschließenden Abschnitt 5, erscheinen die mit der Subsidiaritätsrüge verknüpften Hoffnungen nur in bestimmten Konstellationen berechtigt, die selbst im vermeintlichen „Erfolgsfall“ Monti II nicht gegeben waren.

\section{Die Subsidiaritätskontrolle in der europarechtlichen und politikwissenschaftlichen Debatte}

Insbesondere in der europarechtlichen, aber auch in der politikwissenschaftlichen Literatur ist die Einführung der Subsidiaritätskontrolle mit Interesse begleitet worden. In diesem Abschnitt streifen wir drei der betreffenden Forschungen und Kontroversen, die für unsere Betrachtungen von Relevanz sind, und legen den Schwerpunkt dabei auf die Frage der politischen Nutzbarkeit des neuen Instruments. 
Einige Autorinnen und Autoren diskutierten im Zuge der Einführung der Subsidiaritätskontrolle, ob es sich bei ihr um eine "scharfe Klinge" oder ein "stumpfes Schwert" handelt (siehe etwa Höscheidt 2008; Kiiver 2012; Koch u. Kullas 2010; Pabel 2011; Semmler 2010, S. 532). Eine lebhafte Kontroverse kam hierüber indes nicht in Gang. Vielmehr sind sich die Autorinnen und Autoren darüber einig, dass sich die mit dem neuen Instrument verbundenen Hoffnungen nicht mit einer etwaigen Veto-ähnlichen Schärfe des Verfahrens begründen lassen. Die Subsidiaritätsrüge stattet die nationalen Parlamente mit einer neuen, potenziell öffentlichkeitswirksamen Form von "Voice“ aus. Ist die Kommission aber gewillt, an einem Gesetzesvorhaben festzuhalten, kann sie durch die Rügen nationaler Parlamente nicht daran gehindert werden. ${ }^{6}$ Auch in einem anderen Sinne wurde das neue Verfahren in der begleitenden Literatur als eher "stumpfe Klinge“ gewertet: Die Hürden für die Vergabe der "gelben Karte", so wird angemerkt, lägen recht hoch, und namentlich sei die zur Verfügung stehende Acht-Wochen-Frist zur Formulierung von Rügen angesichts der den Parlamenten abverlangten horizontalen Koordinationsleistung knapp bemessen.

Kontroverser wurde die Frage diskutiert, ob das neue Instrument eng, einer rechtlichen Kompetenzprüfung ähnelnd auszulegen sei oder aber weit und politisch. Wenden wir uns diesen Möglichkeiten im Einzelnen zu. Eine engstmögliche Auslegung der den Parlamenten zugewiesenen Kontrollaufgabe würde ausschließlich danach fragen, ob ein Tätigwerden der europäischen Organe durch eine rechtsförmige Kompetenzgrundlage gedeckt ist. Das wäre eine ultra viresKontrolle, wie sie sich das deutsche Bundesverfassungsgericht vorbehält. Trotz unterschiedlicher Ansichten über die Weite der neuen Kontrollaufgabe scheint weitgehend akzeptiert, dass die Subsidiaritätskontrolle mehr ist als nur eine Kompetenzkontrolle. Ein Subsidiaritätstest setzt einen vorgelagerten Kompetenztest vielmehr voraus, um anschließend prüfen zu können, ob die entsprechende Handlungsermächtigung von der europäischen Ebene im Rahmen der konkurrierenden Gesetzgebung tatsächlich genutzt werden soll. Aus diesem Grund wurde unter Juristen kontrovers diskutiert, ob die Frage einer etwaigen Verletzung des Prinzips der enumerativen Einzelermächtigung überhaupt als Ausgangspunkt des Prüfverfahrens - und daher als legitimer Gegenstand der Befassung durch nationale Parlamente - anzusehen ist (siehe für die Einzelheiten die zahlreiche bei Buschmann u. Daiber 2011, Fn. 15, zitierte Literatur).

Was aber ist die Alternative? Sollen sich die nationalen Parlamente aufgerufen sehen, jedem europäischen Rechtsakt, der ihren Präferenzen irgendwie zuwiderläuft, nunmehr im Namen der Subsidiarität im Frühstadium zu widersprechen? Vor einer derart extensiven Interpretation warnt beispielsweise Becker (2013, S. 29) und spricht sich für eine enge Auslegung der Prüfungsaufgabe aus. Kann es eine enge, an quasi-objektiven Kriterien orientierte und politische Prüfungskriterien konsequent ausschließende Handhabung des neuen Kontrollinstruments

6 Besonders weit geht in dieser Hinsicht ein Reformvorschlag von Chalmers (2013, S. 9-10): Die Kommission solle einen Richtlinien- oder Verordnungsvorschlag nur dann an den Rat weitergeben dürfen, wenn zwei Drittel der mitgliedstaatlichen Parlamente dies zuvor ausdrücklich gebilligt haben. 
überhaupt geben? In der Literatur finden sich durchaus optimistische Stimmen. Goldoni (2014) argumentiert, eine Beschränkung der Subsidiaritätskontrolle auf einen national insufficiency test und einen comparative efficiency test sei möglich. Koch und Kullas (2010, S. 18-20) buchstabieren aus, wie eine an objektiven ordnungspolitischen Kriterien orientierte Subsidiaritätskontrolle aussehen könnte. Die Frage müsse lauten, ob die Effizienzgewinne einer europaweiten Koordination deren Kosten übersteigen. Effizienzgewinne seien durch Prüfung der Internalisierung externer Effekte sowie der Skalenerträge (Größenvorteile) zu erfassen. Als Kosten seien Einbußen an Standortwettbewerb und die Nichtberücksichtigung dezentraler Präferenzen zu erfassen. Übersteigen nun die Gewinne die Kosten, liegt kein Verstoß gegen das Subsidiaritätsprinzip vor.

Aus politikwissenschaftlicher Perspektive erscheint die Möglichkeit einer konsequent politisch entleerten, ausschließlich an mess- und daher objektivierbaren Kriterien orientierten Subsidiaritätskontrolle freilich naiv. $\mathrm{Ob}$ eine Maßnahme „auf Unionsebene besser zu verwirklichen“ ist (so der Wortlaut in Art. 5 Abs. 3 EUV), lässt sich nicht unabhängig von der Frage bewerten, ob sie überhaupt verwirklicht werden soll. Bei jeder Maßnahme, die Verteilungswirkungen entfaltet, werden Repräsentanten unterschiedlicher gesellschaftlicher Gruppen, Länder, Traditionen und Ideologien naturgemäß zu unterschiedlichen Antworten gelangen. Vor diesem Hintergrund darf nicht verwundern, dass "Subsidiarität" in der politischen Praxis als schillernder Kampfbegriff fungiert, der zur Rechtfertigung höchst unterschiedlicher integrationspolitischer Vorstellungen verwendet wird. ${ }^{7}$ Halten wir für die Zwecke der vorliegenden Untersuchung fest, dass weder das Schlagwort der Subsidiarität noch die Nutzung des neuen Kontrollinstruments Aufschluss über die Art der Konflikte geben, in denen Begriff und Instrument in Stellung gebracht werden, und dass die möglichen Anwendungsmöglichkeiten weit über Konflikte über etwaige Verletzungen des Prinzips der enumerativen Einzelermächtigung hinausgehen können.

Von den genannten Debatten zu unterscheiden ist zudem ein empirischer Forschungszweig, der - über unterschiedliche Zeitpunkte und Gesetzesentwürfe hinweg - nach den Determinanten der Bereitschaft der Parlamente fragt, begründete Stellungnahmen zu europäischen Richtlinien- und Verordnungsentwürfen abzugeben. Zwei entsprechende Studien wurden bisher vorgelegt. Breukers (2013) zufolge wirken die Nettozahlerposition eines Landes, die Ländergröße und der Grad an Polarisierung innerhalb der Parlamente begünstigend auf das Rügeverhalten, einen schwach treibenden Einfluss findet Breukers zudem für den Bikameralismus und die Größe der Parlamente. Die Anzahl effektiver Parteien und das Vertrauen der Bürgerinnen und Bürger in die EU scheinen, der Autorin zufolge, keinen Einfluss auf die Häufigkeit von Stellungnahmen auszuüben. Gattermann und Hefftler (2015) identifizieren den Grad an Strittigkeit der europäischen Integrati-

7 Eine eindrückliche Illustration dieses Umstands ist die Tatsache, dass die Parteien CDU, CSU, SPD, Bündnis 90/Grüne, Die Linke und FDP trotz ihrer unterschiedlichen integrationspolitischen Vorstellungen in ihren Wahlprogrammen zur Europawahl 2014 gleichermaßen für eine verbesserte Durchsetzung des Subsidiaritätsprinzips warben bzw. ihre jeweiligen Forderungen mit diesem Schlagwort unterfütterten. 
on innerhalb der Parlamente, die Salienz der betreffenden Gesetzesvorhaben und Minderheitsregierungen als Faktoren, die die Rügebereitschaft der Parlamente begünstigen, während wirtschaftliche Rezessionen die Rügewahrscheinlichkeit zu minimieren scheinen. Keinen Einfluss finden die Autorinnen für die parteipolitische Umstrittenheit der jeweiligen nationalen Integrationspolitik sowie für die Positionierung der maßgeblichen Acht-Wochen-Einspruchsfrist kurz vor nationalen Wahlen. Wir werden an diese Studien in Abschnitt 4 anknüpfen, wenn wir die Verteilung der Rügen über die Parlamente der EU-Mitgliedsländer zur Analyse der Konfliktkonstellation im Fall „Monti II" nutzen.

\section{Die Auseinandersetzung um Monti II}

In diesem Abschnitt wenden wir uns der Geschichte des durch die vorgeschlagene Verordnung aktualisierten Konflikts zu und rücken dabei zwei Aspekte ins Zentrum: die Rolle des EuGH bei der von den rügenden Parlamenten beklagten (etwaigen) Subsidiaritätsverletzung sowie die Mehrdimensionalität der unterliegenden Konfliktstruktur.

Die Vorlage des Kommissionsvorschlags für eine „Monti II“-Verordnung im März 2012 war Teil eines Konflikts, der sich mindestens seit den Süderweiterungen der achtziger Jahre durch die Geschichte der europäischen Integration zieht und dessen Analyse ganze Regale in den juristischen, politik- und wirtschaftswissenschaftlichen Fachbibliotheken füllt. Im Kern dreht sich die Auseinandersetzung um die Reichweite der Binnenmarktregeln gegenüber nationalen Rechtsbeständen, insbesondere gegenüber dem Arbeitskampfrecht und dem Territorialitätsprinzip des Arbeitsrechts (ein Land, ein Arbeitsrecht). Im Dezember 2007 setzte mit den Entscheidungen zu Laval und Viking eine bis heute andauernde Serie von EuGH-Urteilen ein, die in der Öffentlichkeit außergewöhnlich kritisch diskutiert wurde und wird. ${ }^{8}$ In unserem Zusammenhang sind zwei Aspekte von Relevanz (zu den Details Joerges u. Rödl 2009; Rödl 2009, S. 893-894; Sack 2010, S. 620622; Seikel u. Absenger 2015). Erstens, das europäische Höchstgericht setzte der Anwendung nationalen Arbeitsrechts auf im Rahmen der Dienstleistungsfreiheit entsandte Arbeitnehmer durch eine umstrittene Interpretation der Entsenderichtlinie von $1996^{\circ}$ enge Grenzen. In Laval verdeutlichte der EuGH, dass die arbeitsrechtlichen Standards, deren Einhaltung Empfängerländer von entsendenden Unternehmen einfordern dürfen, in Art. 3 (1) der Richtlinie abschließend aufgezählt werden. Jedes Mehr an Übertragung nationaler Standards auf Entsendefirmen verletzt demnach die Dienstleistungsfreiheit des Entsenders (siehe zu diesem Aspekt auch das Nachwort zu diesem Text). Zweitens, der EuGH löste Kollisionen zwischen der Ausübung der europäischen Grundfreiheiten und nationalem Arbeitskampfrecht durch Einordnung des Arbeitskampfs in den auf den Rechtssachen Dassonville, Cassis de Dijon und Gebhard ${ }^{10}$ beruhenden vierstufigen

8 EuGH C-341/05 (Laval) und EuGH C-438/05 (Viking).

9 RL 96/71/EG.

10 EuGH Rs. 8/74 (Dassonville), EuGH Rs. 120/78 (Cassis de Dijon) und EuGH Rs. 120/78 (Gebhard). 
Grundfreiheitentest. Beschränken also Arbeitskämpfe die Ausübung einer Grundfreiheit, befinden sie sich - so die Gebhard-Formel - nur dann im Einklang mit den Binnenmarktregeln, wenn sie in nichtdiskriminierender Weise angewandt werden, aus zwingenden Gründen des Allgemeininteresses gerechtfertigt sind, zur Erreichung des angestrebten Ziels tatsächlich geeignet sind und über das zur Zielerreichung Notwendige nicht hinausgehen. Aus Sicht der Gewerkschaften lief diese Einordnung des Arbeitskampfs in den auf Binnenmarktstörungen angewandten Gebhard-Test auf eine Unterordnung des Streikrechts unter die Grundfreiheiten hinaus, obwohl der EuGH nominell die Gleichrangigkeit von Grundfreiheiten und Grundrechten anerkannte (vgl. zur nominellen Gleichrangigkeit Rn. 91 in Laval und Rn. 44 in Viking; ausführlich zu diesem Problem: Höpner 2016a, S. 248-249). Besonders bemerkenswert ist in diesem Zusammenhang der vom EuGH eingeforderte Einklang der Arbeitskampfziele, d. h. der Arbeitskampfforderungen - nicht lediglich: der Arbeitskampfmittel - mit zwingenden Erfordernissen des Allgemeininteresses.

Die Struktur dieses Konflikts ist komplex und gleichzeitig typisch für Integrationskonflikte, bei denen supranationale marktschaffende Regeln mit mitgliedstaatlichen, potenziell marktbeschränkend wirkenden Rechtsbeständen kollidieren (vgl. grundlegend zur Mehrdimensionalität von Integrationskonflikten Hooghe u. Marks 1999). Welche Beweggründe könnten nationale oder transnationale Parteien und Organisationen, Unternehmen, Einzelpersonen oder Regierungen haben, den im Zuge der europäischen Integration schon lange schwelenden und durch die 2007 einsetzende Serie an EuGH-Urteilen aktualisierten Konflikt unterschiedlich gelöst sehen zu wollen? Mindestens drei Konfliktdimensionen lassen sich unterscheiden: eine um die Reichweite der Integration, eine zwischen Links und Rechts und eine zwischen Ländergruppen.

1. Eine Konfliktdimension zwischen Befürwortern nationaler Autonomie und Befürwortern weitreichender europäischer Integration. Je größer der Anwendungsbereich der europäischen Grundfreiheiten, umso mehr an nationalen Rechtsbeständen wird durch das Europarecht tangiert, transformiert oder gar außer Kraft gesetzt. Jedes Mehr an Verdrängung nationaler Rechtsbestände durch europäisches Recht - hier: durch die Binnenmarktregeln - ist ein Mehr an europäischer Integration. Präferenzträger mit ausgeprägter Intcgrationsskepsis könnten daher versucht sein, den Anwendungsbereich der europäischen Grundfreiheiten gegenüber nationalem Recht eng begrenzt sehen zu wollen, während Präferenzträger mit ausgeprägter Integrationsbefürwortung für weniger Autonomieschutz eintreten könnten.

2. Eine Konfliktdimension zwischen Links und Rechts. Linke Parteien stehen Gewerkschaften näher als rechte Parteien, und es waren vor allem Gewerkschaften, die ihre Errungenschaften durch die vom EuGH vorgegebene Interpretation der Entsenderichtlinie sowie den Umgang mit Kollisionen zwischen Grundfreiheiten und Tarifautonomie in Gefahr sahen. Eher linke Personen und Organisationen könnten einer Erfassung des Streikrechts durch die Binnenmarktregeln daher skeptischer gegenüberstehen als eher konservative Personen und Organisationen. 
3. Eine Konfliktdimension zwischen Ländergruppen. Entsendeströme verlaufen vor allem von den ärmeren, hinsichtlich ihrer tarifrechtlichen Standards schwächer regulierten Ökonomien im Süden und im Osten der EU zu den reicheren, mit strikteren tarifrechtlichen Standards ausgestatteten Ökonomien in der Mitte und im Norden der EU. Je enger der Katalog an Standards, der auf entsandte Beschäftigte übertragen oder mittels Arbeitskampf durchgesetzt werden darf, umso besser für die Unternehmen der Entsendeländer, während die Empfängerländer Abstriche bei der Territorialität ihres Arbeitsrechts sowie Unterbietungskonkurrenz hinnehmen müssen (Eichhorst 2000). Präferenzträger aus den Hochlohnökonomien mit vergleichsweise striktem tariflichem Arbeitnehmerschutz könnten der Erfassung des Arbeits- und Streikrechts durch die Grundfreiheiten daher skeptischer gegenüberstehen als Präferenzträger aus den ärmeren Ländern mit schwächer entwickeltem Arbeitnehmerschutz.

„Monti II" ist eine direkte Folge der durch Laval und Viking aktualisierten Konflikte. Der Europäische Gewerkschaftsbund reagierte auf die Urteile mit scharfem Protest und Forderungen nach einer Überarbeitung der Entsenderichtlinie sowie einer Höhergewichtung des Streikrechts bei Abwägungen gegen die Grundfreiheiten. ${ }^{11}$ Der Tendenz nach schloss sich auch das EP diesen Forderungen an: Die Kommission solle, so eine vom Oktober 2008 datierende EP-Entschließung, die Entsenderichtlinie prüfen und gegebenenfalls überarbeiten. ${ }^{12} \mathrm{Im}$ September 2009 kündigte Kommissionspräsident Barroso an, im Falle seiner Wiederwahl auf eine Lösung der durch die EuGH-Urteile aufgeworfenen bzw. aktualisierten Konflikte hinwirken zu wollen. ${ }^{13}$ Im März 2012 legte die Kommission dann - neben einem im Mai 2014 verabschiedeten Vorschlag für eine Durchsetzungsrichtlinie zur Entsenderichtlinie, den wir an dieser Stelle nicht weiter vertiefen - einen Vorschlag für eine „Verordnung des Rates über die Ausübung des Rechts auf Durchführung kollektiver Maßnahmen im Kontext der Niederlassungs- und der Dienstleistungsfreiheit" vor. ${ }^{14}$ Der Kurzname "Monti II" rührt von einem Bericht von Mario Monti (2010) sowie von der Anknüpfung an die so genannte "Monti-Klausel“ einer älteren Verordnung her, in der es um das Streikrecht im Kontext der Warenverkehrsfreiheit ging. ${ }^{15}$

Anders als von den Gewerkschaften und weiten Teilen des EPs erhofft, enthielt der Vorschlag keine Höhergewichtung des Streikrechts gegenüber den Grundfreiheiten oder etwa - was denselben Effekt gehabt hätte - eine Ausnahme des kollektiven Arbeitsrechts vom Anwendungsbereich der Grundfreiheiten. Vielmehr zielte der Vorschlag auf eine Kodifizierung des seit Viking und Laval ergangenen

11 Siehe die vom 04.03.2008 datierende Entschließung des Exekutivausschusses des Europäischen Gewerkschaftsbunds: http://www.etuc.org/sites/www.etuc.org/files/ResolutionDE-2_2.pdf (Zugriff: 08.06.2016).

12 Entschließsung 2008/2085(INI).

13 Rede von Barroso vor dem EP am 15.09.2009, abrufbar unter: http://europa.eu/rapid/pressrelease_SPEECH-09-391_en.htm_(Zugriff: 08.06.2016).

$14 \operatorname{COM}(2012) 130$ final, 2012/0064(APP).

15 Verordnung Nr. 2679/98. 
Richterrechts durch den europäischen Gesetzgeber. Namentlich sei es auf Grundlage der Verordnung Aufgabe der Arbeitsgerichte, zu prüfen, „ob die betreffenden [Arbeitskampfm]aßnahmen Ziele verfolgen, die ein berechtigtes Interesse darstellen ". ${ }^{16}$ Neben dieser Kodifizierung sah Art. 4 des Verordnungsvorschlags ein Frühwarnsystem für Streikmaßnahmen mit transnationalen Auswirkungen vor: Im Falle absehbarer grenzüberschreitender Auswirkungen von Arbeitskämpfen seien unverzüglich etwaig betroffene Mitgliedstaaten sowie die Kommission zu informieren. Weil Art. 153(5) AEUV das Arbeitskampfrecht ausdrücklich vom Kompetenzkatalog der europäischen Organe ausnimmt, stützte sich der Vorschlag auf die manchmal als „Abrundungskompetenz" bezeichnete Flexibilitätsklausel des Art. 352 AEUV. Diese Klausel ermöglicht die Aktivierung der europäischen Gesetzgebung, wenn ein von den Verträgen vorgegebenes Ziel verfolgt wird, die hierfür notwendige Kompetenznorm aber fehlt. Auf Art. 352 AEUV gestützte Gesetzesakte bedürfen der einstimmigen Zustimmung des Rats.

An dieser Stelle schließt sich der Kreis zum neuen Kontrollverfahren. „Monti II" erhielt innerhalb der achtwöchigen Frist Subsidiaritätsrügen von zwölf nationalen Parlamenten. Die begründeten Stellungnahmen kamen von Parlamenten aus Belgien, Dänemark, Finnland, Frankreich, Großbritannien, Lettland, Luxemburg, Malta, den Niederlanden, Polen, Portugal und Schweden. Unter den rügenden Parlamenten waren sieben Einkammerparlamente (14 Gegenstimmen) sowie fünf Kammern aus Zweikammersystemen (5 Gegenstimmen). Nur im Falle Frankreichs rügte eine zweite Kammer den Verordnungsvorschlag, alle anderen Rügen aus Ländern mit Bikameralismus kamen von ersten Kammern. Mit den insgesamt 19 von 18 notwendigen Gegenstimmen wurde im Mai 2012 im Ergebnis erstmals die Schwelle an begründeten Stellungnahmen überschritten, die für den Prozess der "gelben Karte" notwendig war. Das bedeutete konkret, dass die Kommission aufgefordert war, ihren Vorschlag zu überprüfen. Anfang Juni 2012 gab die Kommission die Überschreitung des Schwellenwerts bekannt. Sie unterzog ihren Vorschlag einer erneuten Prüfung und kam zu dem Ergebnis, dass keine Verletzung des Subsidiaritätsprinzips erkennbar war. Dennoch beschloss sie im September 2012, ihren Vorschlag zurückzuziehen, da die für die Verordnung notwendige Unterstützung nicht erreichbar erschien. ${ }^{17}$

Inwieweit lassen sich die Vorgänge nun als Beispiel für einen durch das neue Verfahren effektuierten Subsidiaritätsschutz, also als Beleg für die Berechtigung der ersten der eingangs vorgestellten Hoffnungen werten? Die „gelbe Karte" war, so ließe sich ja durchaus folgern, trotz der eine Verletzung des Subsidiaritätsprinzips verneinenden Stellungnahme der Kommission erfolgreich. Den nationalen Parlamenten sei es gelungen, Druck auf die Kommission auszuüben und die europäische Gesetzgebung zu beeinflussen, schreibt Boronska-Hryniewiecka (2013, S. 177); "national parliaments have the capacity and willingness to use their new powers to exercise a collective influence in EU affairs", fasst Cooper (2015, im Abstract) die Ergebnisse seiner Fallbetrachtung zu Monti II zusammen. Tatsäch-

$16 \operatorname{COM}(2012) 130$ final, 2012/0064(APP), S. 11.

17 Siehe die Einzelheiten in $\operatorname{COM}(2013) 566$ final sowie im vom 12.09 .2012 datierenden und mit der Kennung Ares(2012)1058907 versehenen Antwortbrief der Kommission. 
lich aber muss diese Deutung angesichts der hier nachgezeichneten Abläufe zurückgewiesen werden. Denn nicht nur führte die "gelbe Karte“ zu einem Ergebnis, zu dem der europäische Gesetzgebungsprozess angesichts der Abwehrhaltung zahlreicher Länder einerseits und der notwendigen Einstimmigkeit im Rat andererseits ohnehin gelangt wäre. Auch bewirkte das Scheitern der Verordnung lediglich einen Stopp der Kodifizierung einer Rechtsprechung, die mit oder ohne Kodifizierung Bestand hatte und weiter hat. In Bezug auf den Subsidiaritätsschutz hatte das neue Instrument daher keinen erkennbaren Mehrwert erzeugt.

\section{Ländervergleichende Analyse}

In diesem Abschnitt nehmen wir die Lage der den Fall „Monti II“ dominierenden Konfliktlinie eingehender unter die Lupe, um zu prüfen, ob das neue Kontrollinstrument einen Beitrag zur Stärkung der Kohärenz der europäischen Parteienfamilien leisten konnte. Zu diesem Zweck analysieren wir, entlang welcher Merkmale sich die Gruppe der 12 Länder, deren Parlamente eine Rüge gegen die vorgeschlagene „Monti II“-Verordnung aussprachen, von der Gruppe der restlichen 15 Mitgliedstaaten unterscheidet, die innerhalb der maßgeblichen Acht-Wochen-Frist keine Rügen aussprachen. ${ }^{18}$ Anders ausgedrückt interessiert uns die Frage, ob es Prädiktoren gibt, anhand derer sich das Rügeverhalten der beteiligten Länder erklären lässt. Zwar ist von vornherein davon auszugehen, dass das entsprechende Verhalten der mitgliedstaatlichen Parlamente von zahlreichen idiosynkratischen Faktoren geprägt wurde: Beispielsweise wurde eine frühzeitige Beschäftigung mit dem Kommissionsvorschlag in Irland durch das anstehende Referendum zum Fiskalpakt gehemmt, in der französischen Assemblée Nationale lag die Arbeit aufgrund des Präsidentschaftswahlkampfs und der nachfolgenden Parlamentswahl brach (die zweite Kammer, der französische Senat, sprach hingegen eine Rüge aus). Aus Tschechien und vom deutschen Bundesrat kamen Stellungnahmen, die inhaltlich Rügen gleichkamen, aber zu spät abgegeben wurden, um als formelle Rügen zu zählen. ${ }^{19} \mathrm{Da}$ wir aber keinen Grund für die Annahme haben, dass dieser "Noise“ die Daten systematisch in eine Richtung verzerrt, gehen wir davon aus, dass etwaige kausale Bestimmungsfaktoren des Rügeverhaltens durch die ländervergleichende Methode gleichwohl aufgedeckt werden können.

Nachfolgend vergleichen wir Mittelwerte der uns interessierenden Ländervariablen und geben auf Grundlage eines t-Tests an, ob sich die errechneten Mittelwerte in signifikanter Weise voneinander unterscheiden. Um die Zugänglichkeit der Analyse nicht über Gebühr einzuschränken, verzichten wir darauf, in getrennten Schritten zunächst formale Hypothesen zu formulieren, dann die Operationalisierungen vorzustellen und schließlich die Ergebnisse zu präsentieren. Stattdessen gehen wir Variable für Variable vor und handeln Hypothese, Operationalisierung und Befund in jeweils einem Gesamtschritt ab. Was die Reihenfolge der Variablen betrifft, orientieren wir uns an der Abfolge der drei in Abschnitt 3

18 Weil Kroatien erst im Jahr 2013 Mitglied der EU wurde, beruht die Analyse auf der EU der 27 (statt 28) Mitglieder.

19 Siehe zu diesen Beispielen Becker (2013, S. 25) und Cooper (2015, S. 1418). 
unterschiedenen Konfliktdimensionen. Wir wenden uns daher zunächst Indikatoren zum Euroskeptizismus zu, gehen dann zu einem Indikator der Links-RechtsKomposition der Regierungen über und behandeln im Anschluss Indikatoren zum Wohlstandsniveau und zum tariflichen Regulierungsniveau der beteiligten Länder. Ein Überblick über die Ergebnisse findet sich in Tabelle 1.

Tabelle 1: Merkmale der Länder mit und ohne Subsidiaritätsrüge gegen den Vorschlag für eine „Monti II"-Verordnung (Mittelwerte)

\begin{tabular}{|l|c|c|c|}
\hline Variable & $\begin{array}{c}\text { Rüge } \\
\text { (12 Lander) }\end{array}$ & $\begin{array}{c}\text { keine Rüge } \\
\text { (15 Lander) }\end{array}$ & $\mathrm{t}$-Wert \\
\hline $\begin{array}{l}\text { Vertrauen der Bürgerinnen und Bürger in } \\
\text { EU-Institutionen (\%) }\end{array}$ & 48 & 43 & 1,20 \\
$\begin{array}{l}\text { Zufriedenheit der Bürgerinnen und Bürger mit der } \\
\text { EU-Demokratie (\%) }\end{array}$ & 51 & 44 & 1,70 \\
$\begin{array}{l}\text { Einschätzung der Bürgerinnen und Bürger zu } \\
\text { Zukunftsaussichten durch EU-Mitgliedschaft (\%) }\end{array}$ & 61 & 59 & 0,43 \\
$\begin{array}{l}\text { Einwohnerzahl (in Mio.) } \\
\text { Parteipolitische Zusammensetzung der Regierungen } \\
\text { (1= rechts, 5 = links) }\end{array}$ & 19,0 & 18,2 & 0,10 \\
Parlamentarismus vs. Präsidentialismus & 0,73 & 2,33 & 0,81 \\
(0= Parlamentarismus, 4 = Präsidentialismus) & 27,4 & 16,3 & $2,26^{* *}$ \\
Reales BmP pro Kopf (in Tsd. $€$ ) & 37 & 21 & $2,09 *$ \\
Gewerkschaftlicher Organisationsgrad (\%) & 69 & 52 & $1,72^{*}$ \\
\hline Tarifvertragliche Deckungsrate (\%)
\end{tabular}

Anmerkungen: "* $p<0.05 ;$ * $p<0,1$. Definitionen und Quellen der Variablen: siehe Angaben im Text.

Quelle: Eigene Darstellung.

Wir haben gesehen, dass die Auseinandersetzung um „Monti II“ die Konfliktlinie zwischen dem Schutz nationaler Autonomie und weitgehender europäischer Integration tangierte. Haben daher insbesondere Parlamente aus Ländern mit ausgeprägter Integrationsskepsis Rügen ausgesprochen? Auf den ersten Blick erscheint diese Vermutung nicht unplausibel. So gelten Großbritannien und Dänemark als vergleichsweise integrationsskeptische Länder, und in der Tat finden wir beide Länder in der Gruppe der rügenden Mitgliedstaaten. Zur Messung der Integrationsfreundlichkeit der Bevölkerungen greifen wir auf Daten des Eurobarometers zurück und verwenden dabei die der Rügefrist nächstgelegene Erfassungswelle. Im Standard Eurobarometer 78 vom Herbst 2012 zielten drei Fragen auf die grundsätzlichen Haltungen der Bevölkerungen zur EU: die Fragen über das Vertrauen in die Institutionen der EU, über die Zufriedenheit mit der Demokratie in der EU und über die Zukunftsaussichten des jeweiligen Landes mit oder ohne 
EU-Mitgliedschaft. ${ }^{20}$ Wie nicht anders zu erwarten, sind die Antworten der Befragten im Ländervergleich positiv korreliert (vgl. die Korrelationsmatrix im Anhang). Es zeigt sich, dass sich die Bevölkerungen der Ländergruppen mit und ohne Rüge hinsichtlich ihres Integrationsvertrauens bzw. ihres EU-Skeptizismus nicht signifikant unterscheiden. Wir haben also keine Gründe für die Annahme, das Rügeverhalten der Länder sei vor allem von der Integrationsaversion der jeweiligen Bevölkerungen getrieben worden. ${ }^{21}$

Zusätzlich wollten wir wissen, ob Rügen besonders von kleinen Ländern kamen. Konflikte zwischen kleinen und großen Ländern können in der europäischen Politik beispielsweise entstehen, wenn große Länder Vertragsrevisionen oder ihre Haltungen zu vorgeschlagenem Sekundärrecht vorverhandeln, den kleinen Ländern anschließend hingegen nur noch die Möglichkeiten der Zustimmung oder Ablehnung verbleiben, ohne noch Details beeinflussen zu können. Die Integrationsskepsis und folglich auch die Rügebereitschaft der Parlamente kleiner Länder könnten daher überdurchschnittlich ausgeprägt sein. Allerdings ließe sich mit ähnlicher Plausibilität auch eine entgegengesetzte Hypothese formulieren: Parlamente großer Länder dürften mit mehr Ressourcen ausgestattet sein und könnten dem Aufwand einer stetigen Beobachtung und Bewertung europäischer Gesetzesvorhaben daher eher gewachsen sein als Parlamente kleiner Länder (in diese Richtung deutet auch ein Ergebnis bei Breukers 2013, S. 42). Zur Messung der Ländergrößen greifen wir auf Einwohnerzahlen aus dem Jahr 2012 zurück. ${ }^{22}$ Wie der Eintrag in Tabelle 1 zeigt, findet sich im Rügeverhalten der untersuchten Länder kein Größeneffekt: Die durchschnittlichen Einwohnerzahlen der beiden Gruppen unterscheiden sich nicht signifikant voneinander.

$\mathrm{Zu}$ einem letzten Test im Zusammenhang mit grundsätzlichen Erwägungen zum Integrationsskeptizismus, der im Prinzip für das Rügeverhalten der Parlamente im vorliegenden Fall verantwortlich sein könnte, leitet uns folgende Überlegung an: In der EU koexistieren parlamentarische Demokratien, semi-präsidentielle politische Systeme und Präsidialsysteme (allerdings ist allein Zypern dem Typus der reinen Präsidialsysteme zuzuordnen). Die Bereitschaft zur Rüge könnte in Präsidialsystemen, in denen die Leitung der Exekutive nicht aus dem Parlament hervorgeht, besonders ausgeprägt sein. Zur Überprüfung greifen wir auf den auf Lijphart (2012, S. 105-129) zurückgehenden fünfstufigen Parlamentarismus-

20 Standard Eurobarometer 78, Herbst 2012, Die öffentliche Meinung in der Europäischen Union. Die drei Fragen lauteten: QA16.1+2+3: „Sagen Sie mir bitte fïr jede dieser europäischen Einrichtungen, ob Sie ihr eher vertrauen oder eher nicht vertrauen: Dem Europäischen Parlament; der Europäischen Kommission; dem Rat der Europäischen Union. “ Als Indikator für das Integrationsvertrauen verwenden wir einen Durchschnittswert der Kategorie „eher vertrauen" für die drei Teilfragen. QA19b: "Sind Sie mit der Art und Weise, wie die Demokratie in der EU funktioniert, alles in allem gesehen, zufrieden? Sagen Sie es mir bitte anhand dieser Skala: „zufrieden“, „nicht zufrieden“. Als Indikator für das Integrationsvertrauen verwenden wir den Anteil der mit "zufrieden" Antwortenden. QA20a.11: „In wieweit stimmen Sie [...] der folgenden Aussag[e] zu oder nicht? (UNSER LAND) wäre besser für die Zukunft gerüstet, wenn es nicht Mitglied der EU wäre." Als Indikator für das Integrationsvertrauen verwenden wir den Anteil der mit „stimme nicht zu“ Antwortenden.

21 Dieses Ergebnis passt zu einem allgemeineren Befund von Auel et al. (2015), die zeigen, dass sich das Ausmaß an parlamentarischer Beschäftigung mit EU-Angelegenheiten (über die Subsidiaritätsrüge hinaus) nicht durch die Integrationsskepsis der jeweiligen Bevölkerungen erklären lässt.

22 Datenquelle: Eurostat. 
Präsidentialismus-Indikator zurück, bei dem 0 für reinen Parlamentarismus steht, 4 für reinen Präsidentialismus und die übrigen Werte für Zwischenstufen. ${ }^{23}$ Es zeigt sich aber, dass auch diese Überlegung nicht zur Aufklärung des Rügeverhaltens im betrachteten Einzelfall beiträgt: Die Indexwerte der Länder mit und ohne Rügen unterscheiden sich abermals nicht signifikant voneinander.

Wie in Abschnitt 3 diskutiert, tangierte der Konflikt um "Monti II" auch die Konfliktdimension zwischen Links und Rechts. Qualitative Einsichten in ausgewählte Fälle stützen den Anfangsverdacht, dass die Debatten um den Verordnungsvorschlag vor allem durch einen Links-Rechts-Gegensatz strukturiert wurden. So weist beispielsweise Becker (2013, S. 26) darauf hin, dass es im Arbeits- und Sozialausschuss des Deutschen Bundestages zu einer parteipolitischen Frontstellung kam, in deren Rahmen die damaligen bürgerlichen Regierungsparteien den maßgeblich von den Gewerkschaften initiierten Protest gegen „Monti II" nicht mittragen wollten.

Kamen die Rügen also vor allem aus linksregierten Ländern? Anders als bei den anderen Hypothesen betrachten wir hier ausschließlich die ersten Parlamentskammern. ${ }^{24}$ Zur Operationalisierung greifen wir auf den von Manfred G. Schmidt entwickelten Index der parteipolitischen Zusammensetzung von Regierungen zurück. ${ }^{25}$ Entgegen der Erwartung zeigt sich kein systematischer Zusammenhang zwischen der parteipolitischen Zusammensetzung der Regierungen und dem Rügeverhalten: Die durchschnittlichen parteipolitischen Verortungen der Regierungen der beiden Ländergruppen unterscheiden sich nicht signifikant voneinander. ${ }^{26}$ Zwar gibt es unter den beobachteten Fällen Länder wie das linksregierte Dänemark (Rüge) und bürgerlich regierte Länder wie Deutschland, Spanien und Tschechien (keine Rüge), auf die die Hypothese passt. Gleichzeitig finden sich aber sowohl linksregierte Länder wie Zypern, Slowenien und die Slowakei, die sich einer Rüge enthielten, als auch bürgerlich regierte Länder wie Großbritannien, Portugal und Lettland, deren Parlamente „Monti II" als Verstoß gegen das Subsidiaritätsprinzip rügten. Im Schnitt waren - entgegen der Hypothese - Länder mit Rüge gegen den Kommissionsvorschlag sogar etwas bürgerlicher bzw. konservativer regiert als Länder ohne Rüge.

23 Datenquelle: Armingeon et al. (2015).

24 Frankreich ist das einzige Land, für das diese Betrachtungsweise einen Unterschied macht (weil nur in Frankreich eine Rüge von einer zweiten Kammer ausgesprochen wurde). Für den Befund macht die Klassifikation Frankreichs keinen Unterschicd: Auch wenn man Frankreich der rügenden Gruppe zuordnet, zeigt sich kein signifikanter Zusammenhang zwischen Regierungszusammensetzung und Rügeverhalten.

25 Maßgeblich ist die parteipolitische Zusammensetzung der Regierungen während der Rügefrist. Der Index hat fünf Stufen: 1 = Vorherrschaft bürgerlicher bzw. konservativer Parteien; 2 = Dominanz bürgerlicher bzw. konservativer Parteien; $3=$ Gleichgewicht zwischen den Blöcken; 4 = Dominanz sozialdemokratischer bzw. linker Parteien; $5=$ Vorherrschaft sozialdemokratischer bzw. linker Parteien. Datenquelle: Armingeon et al. (2015). Die italienische Technokratenregierung haben wir als Mitte-Regierung klassifiziert.

26 Wir haben auch überprüft, ob das Ergebnis durch die Minderheitsregierungen in Bulgarien, Dänemark, den Niederlanden, Rumänien, Schweden und Zypern verzerrt wird. Das ist nicht der Fall. Von den sechs Ländern mit Minderheitsregierungen gingen drei Rügen aus, drei Länder haben nicht gerügt; sowohl unter den drei Ländern mit und ohne Rüge gab es gleichermaßen jeweils eine Linksregierung und zwei bürgerliche bzw. konservative Regierungen. 
Als dritte Konfliktdimension hatten wir in Abschnitt 3 unterschiedlich gelagerte Interessen von Ländergruppen identifiziert, die sich hinsichtlich ihrer Wohlstandsniveaus und tariflichen Schutzstandards unterscheiden. Aufgrund der Struktur des innereuropäischen Wohlstandsgefälles verlaufen die Entsendeströme vor allem von Süd und Ost nach Nord und ins Zentrum der EU. Je restriktiver die Übertragbarkeit von Arbeitsstandards auf aus anderen Ländern entsandte Beschäftigte, umso mehr Konkurrenz einheimischer Firmen durch Entsendefirmen und umso mehr Untergrabung der Einheitlichkeit von Arbeitsstandards ist aufseiten der Zielländer zu erwarten. Der Anreiz zur Rüge, so ließe sich folgern, sollte daher für die wohlhabenden und mit vergleichsweise starken Gewerkschaften und strikten Tarifstandards ausgestatteten Länder Zentral- und Nordeuropas besonders ausgeprägt sein. Wie Tabelle 1 zeigt, wird diese Erwartung in Bezug auf den Wohlstand, gemessen anhand des realen BIP pro Kopf im Jahr 2012, bestätigt. ${ }^{27}$ Das mittlere Wohlstandsniveau der Länder, deren Parlamente sich für eine Rüge entschieden, übersteigt das mittlere Wohlstandsniveau der übrigen Länder sogar erheblich (rund 27.000 Euro im Vergleich zu rund 16.000 Euro).

Ähnliche, die formulierten Erwartungen bestätigende Ergebnisse kommen für Indikatoren zur Gewerkschaftsstärke und zur tariflichen Regulierungsintensität zustande. Mit 37 Prozent erweist sich der durchschnittliche gewerkschaftliche Organisationsgrad der Länder, die eine Subsidiaritätsrüge gegen „Monti II “ aussprachen, als signifikant höher als der entsprechende Organisationsgrad der übrigen Länder, der nur 21 Prozent beträgt. ${ }^{28}$ Und auch der Anteil an Beschäftigten, die von Tarifverträgen erfasst werden, unterscheidet sich zwischen den beiden Ländergruppen in signifikanter Weise. Er beträgt durchschnittlich 69 Prozent in den Ländern, deren Parlamente „Monti II" rügten, aber nur 52 Prozent in den Ländern, die sich nicht zu einer Rüge entschlossen.

Der Konflikt um „Monti II“, so das Ergebnis, scheint vor allem als Konflikt zwischen Ländern mit unterschiedlichen Wohlstands- und Regulierungsniveaus ausgetragen worden zu sein. Die Darstellung in Abbildung 1 illustriert diese Sortierung des Rügeverhaltens entlang von Ländergruppen. In ihr unterscheiden wir im Anschluss an die „Spielarten des Kapitalismus"-Forschung fünf Gruppen europäischer Länder, die sich hinsichtlich der institutionellen Ausgestaltungen ihrer Arbeitsbeziehungen (sowie weiterer Merkmale ihrer Produktionsregime) unterscheiden (Amable 2003; Hall u. Soskice 2001; Nölke u. Vliegenthart 2009): eine sozialdemokratisch-nordische, eine konservativ-mitteleuropäische, eine liberale, eine mediterrane und eine postsozialistisch-osteuropäische Ländergruppe. Für alle Gruppen zeigen die Balken die Anzahlen an Ländern mit und ohne Rüge gegen „Monti II". Wie sich der Grafik entnehmen lässt, waren es vor allem die Parlamente der nord- und mitteleuropäischen „koordinierten " Ökonomien, die sich zur Subsidiaritätsrüge entschlossen. Die liberale Gruppe ist lediglich mit zwei Ländern besetzt, von denen Großbritannien eine Rüge aussprach, Irland aber nicht. In den südlichen und östlichen Ländergruppen sprachen die Länder mehrheitlich keine Rügen aus.

27 Datenquelle: Eurostat.

28 Datenquelle: Visser 2015. 


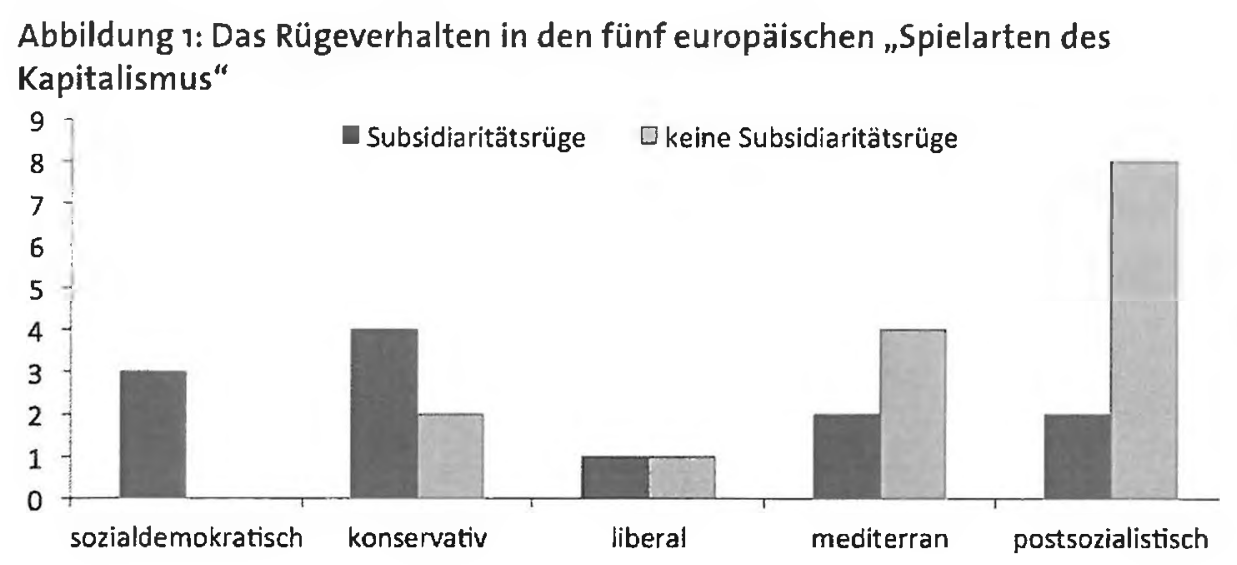

Sozialdemokratisch-nordisch: Dänemark, Finnland, Schweden

Konservativ-mitteleuropäisch: Belgien, Frankreich, Luxemburg, Niederlande, Deutschland, Österreich

Liberal:

Großbritannien, Irland

Mediterran:

Malta, Portugal, Griechenland, Spanien, Italien, Zypern

Postsozialistisch-osteuropäisch: Lettland, Polen, Bulgarien, Tschechien, Estland, Litauen, Ungarn, Rumänien, Slowenien, Slowakei

Quelle: Eigene Darstellung.

Halten wir fest: Mehr als zwischen Autonomie und Integration oder zwischen Links und Rechts manifestierte sich der Konflikt um „Monti II“ offenbar als Frontstellung zwischen Ländergruppen, die mit unterschiedlichen Wohlstandsniveaus ausgestattet sind und die unterschiedlichen "Spielarten des Kapitalismus“ angehören. Diese Einsicht relativiert die Berechtigung der Hoffnung auf eine vom neuen Kontrollinstrument ausgehende Schärfung der Kohärenz der europäischen Parteienfamilien. Denn offensichtlich kann die Strukturierung der Konflikte, bei denen die Subsidiaritätsrüge zur Anwendung kommt, durch den Links-RechtsGegensatz nicht unbesehen vorausgesetzt werden.

\section{Interpretation}

In diesem Abschnitt führen wir die Befunde zusammen, diskutieren ihre Implikationen für die in Abschnitt 1 vorgestellten, mit der parlamentarischen Subsidiaritätskontrolle verknüpften Hoffnungen und wenden uns zunächst der Hoffnung auf einen verbesserten Subsidiaritätsschutz zu. Überdenkt man diese Hoffnung auf Grundlage der vorliegenden Fallbetrachtung, dann zeigt sich, dass ihr eine unhinterfragte Prämisse zugrunde liegt: die Annahme nämlich, Verstöße gegen die Subsidiarität gingen zuvörderst von der europäischen Gesetzgebung aus. Denn nur auf diese zielt das neue Verfahren. Geht der vermeintliche Subsidiaritätsver- 
stoß hingegen vom Europäischen Gerichtshof aus, kann das neue Verfahren den Autonomieschutz nicht effektuieren. Genau dieser Effekt trat im Fall „Monti II“ ein. Ein Erfolg aufseiten der mitgliedstaatlichen Parlamente ließe sich allenfalls im Hinblick auf die in Art. 4 des Kommissionsvorschlags angedachte Meldepflicht für Arbeitskämpfe mit transnationalen Auswirkungen behaupten. Das Gros des Richtlinienvorschlags zielte aber auf die Kodifizierung der vom EuGH vorgenommenen Einordnung des Streikrechts in den vierstufigen Grundfreiheiten-Test. Mit oder ohne sekundärrechtliche Kodifizierung hat diese EuGH-Rechtsprechung Bestand und muss von den nationalen Arbeitsgerichten umgesetzt werden.

Die Hoffnung auf mehr Subsidiaritätsschutz ist also dahingehend zu qualifizieren, dass sie nur in bestimmten Fällen berechtigt ist. Die vorliegende Einzelfallbetrachtung erlaubt keine Aussage über die Struktur der Grundgesamtheit aller Integrationskonflikte, bei denen die Subsidiaritätsrüge im Prinzip zur Anwendung kommen könnte. Aber immerhin lässt sich festhalten, dass die Annahme, Subsidiaritätsverletzungen gingen zuvörderst von der europäischen Gesetzgebung aus, nicht im Einklang mit der politikwissenschaftlichen Integrationsforschung steht. Denn die politikwissenschaftliche Literatur charakterisiert das politische System der EU als mit hohen formellen und informellen Konsenshürden ausgestattet, mit institutionellen Vetopunkten durchzogen und entsprechend schwerfällig (Hix 2008, S. 589-594; Scharpf 1985; Scharpf 1999, S. 70-80; Tsebelis u. Garrett 2001). Wann immer europäische Gesetzesvorhaben transnationale Verteilungswirkungen oder asymmetrisch verteilte institutionelle Anpassungslasten hervorbringen, fällt es den Teilnehmern an der europäischen Integration schwer, tragfähige Kompromisse zu finden. In die europäische Gesetzgebung sind, mit anderen Worten, bereits effektive Mechanismen zum Schutz von Autonomie und Souveränität eingebaut. Insofern ist zunächst einmal zu konstatieren, dass die Subsidiaritätsrüge dem politischen System der EU einen weiteren - potenziell redundanten - unter ohnehin schon zahlreichen Vetopunkten hinzufügt.

Betrachtet man das neue Instrument aus dieser Pcrspektive, dann erscheint auch die äußerst geringe Anzahl von bisher (lediglich) drei erfolgreich gezückten „gelben Karten“" wenig verwunderlich. Wie in Abschnitt 2 dargestellt, wurden in der europarechtlichen und der politikwissenschaftlichen Fachdiskussion die hohen Hürden thematisiert, die der Subsidiaritätsrüge - etwa in Form der knapp bemessenen Acht-Wochen-Frist - aufliegen. Diese Hürden mögen zur geringen Anzahl erfolgreicher Subsidiaritätsrügen beigetragen haben. Mindestens ebenso plausibel erscheint aber eine Alternativhypothese: Das Instrument zielt auf einen Adressaten, von dem fragwürdig ist, ob er für die oftmals beklagten Zentralisierungsdynamiken in der EU verantwortlich zu machen ist. Wenn es sich im Einzelfall als entweder redundant oder wirkungslos erweist, erscheint das relative Desinteresse der Parlamente nur konsequent.

Einen potenziell problematischen Aktivismus lokalisiert die Forschungsliteratur weniger in der europäischen Gesetzgebung, als vielmehr in der „Integration durch Recht “, insbesondere in der weiten Auslegung europäisch geschützter Individualrechte durch die supranationalen Organe Kommission und Europäischer Gerichtshof (Alter 2001; Höpner u. Schäfer 2012; Höreth 2008, S. 191-308; Scharpf 1999, S. 52-69; Scharpf 2009; Schmidt 2012; Weiler 1987). „Monti II“ war ein Ergebnis 
dieser judiziellen Integrationsdynamik und, wie beispielsweise Martinsen und Blomqvist (2009), Schmidt (2009) und Schreinermacher (2014) zeigen, in dieser Hinsicht kein Einzelfall: Viel Sekundärrecht zur Ausgestaltung des Verhältnisses von Binnenmarktfreiheiten und nationalen Regulierungen kodifiziert zuvor ergangene EuGH-Rechtsprechung. Für all diese Fälle muss die Subsidiaritätsrüge, wie bei dem Konflikt um „Monti II“, zwangsläufig ins Leere greifen.

Erinnern wir uns in diesem Zusammenhang auch daran, dass der Subsidiaritätsrüge eine Subsidiaritätsklage an die Seite gestellt ist (vgl. Abschnitt 1): Nach Abschluss eines europäischen Gesetzgebungsakts können nationale Parlamente nunmehr gerichtlich überprüfen lassen, ob ein Verstoß gegen Subsidiarität und Verhältnismäßigkeit vorliegt. Was wäre passiert, wäre die Verordnung beschlossen worden und hätte das dänische Folketinget oder der schwedische Riksdag die beiden wahrscheinlichsten Kandidaten, die auch als erste ihre Rügen gegen „Monti II" formulierten - anschließend geklagt? Dann wäre der EuGH aufgefordert worden, einen Akt der europäischen Gesetzgebung zu verwerfen, der vor allem seine eigene, mittlerweile gefestigte Rechtsprechung kodifizierte. Über die Erfolgsaussichten muss nicht gerätselt werden. Treffender ließe sich kaum illustrieren, wie stumpf das neue Instrument tatsächlich ist, wenn Konflikte um Subsidiarität nicht von der europäischen Gesetzgebung, sondern von der „Integration durch Recht" ausgehen. ${ }^{29}$

Wenden wir uns nun den Hoffnungen zu, die auf eine virtuelle Verdichtung der nationalen Parlamente zu einer "dritten Kammer" auf europäischer Ebene gerichtet sind, auf ein Mehr an Interesse und Diskurs über europäische Themen aufseiten demokratisch legitimierter Instanzen und auf die Entstehung eines neuen Kommunikationskanals zwischen Kommission und mitgliedstaatlichen Legislativen. Wie Goldoni (2014, S. 106) anmerkt, war im Zuge der Auseinandersetzung um „Monti II“ keine Entstehung eines als „dritte Kammer" interpretierbaren kollektiven Agenten zu beobachten, sondern vielmehr eine Aggregation individueller, auf nationalstaatlicher Ebene angesiedelter Präferenzen. Das trifft auf das Einzelereignis gewiss zu, spricht aber nicht gegen die mittel- bis langfristige Berechtigung der Hoffnung. Selbst wenn die Zweifel an der Effektivität des Instruments seine Nutzung durch die nationalen Parlamente hemmen könnte, wird sich die Notwendigkeit der Prüfung der europäischen Gesetzgebung auf eventuelle „Rügefälle" für die nationalen Parlamente in Zukunft immer wieder stellen. Die Erwartung erscheint angemessen, dass sich für die hierfür hilfreiche horizontale Koordination zwischen den zuständigen Parlamentsausschüssen Verfahren und feste Kommunikationskanäle entwickeln (Reiding et al. 2016, S. 100-101), zumal die jüngere Forschung einen generellen Trend des Ausbaus der EU-Aktivitäten nationaler Parlamentsverwaltungen nachweist (speziell zur horizontalen Koordinati-

29 Nichts anderes gilt für andere europäische Instrumente, deren Nutzung weniger hohe Konsenshürden aufliegen als der europäischen Gesetzgebung und die daher ungleich anfälliger für Kollisionen mit dem Autonomiebedürfnis der Mitgliedstaaten sind. Man denke hier an das Mandat der Europäischen Zentralbank, an das neue Verfahren zur Erkennung und Vermeidung makroökonomischer Ungleichgewichte oder an die Beteiligung der europäischen Organe an der Troika bzw., seit ihrer Erweiterung um den Europäischen Stabilitätsmechanismus (ESM), der Quadriga: Sie alle werden yon der Subsidiaritätskontrolle nicht erreicht. 
onsfunktion: Högenauer u. Neuhold 2015, S. 346-348). Inwieweit sich dieses Mehr an koordinierter Beschäftigung mit der europäischen Politik im Sinne einer verbesserten Legitimitätszufuhr an die EU wird interpretieren lassen - und sich tatsächlich in ein verbessertes Legitimitätsempfinden der Bürgerinnen und Bürger wird übersetzen können -, sei unter Vorbehalt zukünftiger Erfahrungen und entsprechender Forschung dahingestellt. Aber eine verstärkte Befassung nationaler Parlamente mit wichtigen europäischen Fragen sowie eine entsprechende horizontale Koordination dürften stattfinden und wären als Gegengewicht zur Exekutivlastigkeit der europäischen Politik zweifellos zu begrüßen (nachdrücklich: Chalmers 2013). Die vorliegende Betrachtung führte zumindest zu keinen Ergebnissen, die einer solchen Erwartung entgegenstehen würden.

Wesentlich skeptischer wäre im Licht von „Monti II" aber die Hoffnung auf einen vertrauensvollen, auf Dauer gestellten, vertikale Deliberation in Gang setzenden Kommunikationskanal zwischen der Kommission und den nationalen Parlamenten zu beurteilen. Der vermeintliche Sieg der Parlamente beruhte nicht darauf, dass die Kommission eine etwaige Verletzung der Subsidiarität eingeräumt oder in ihrem Antwortschreiben auch nur eine Abwägung der von den Parlamenten vorgebrachten Argumente vorgenommen hätte. Vielmehr legte sie kurz und bündig dar, dass sie die Einwände der Parlamente geprüft habe und als gegenstandslos betrachte. ${ }^{30}$ Allein aufgrund der Einsicht in die Kräfteverhältnisse zog die Kommission ihren Verordnungsvorschlag im September 2012 zurück: Weil eine Kompetenznorm zur Regelung des kollektiven Arbeitsrechts fehlte, hatte sie ihr Vorgehen auf Art. 352 AEUV gestützt und war daher auf einen einstimmigen Beschluss des Rats angewiesen. Die Subsidiaritätsrügen aus zwölf Ländern signalisierten der Kommission, dass diese Einstimmigkeit nicht erreichbar war. ${ }^{31} \mathrm{Im}$ Prinzip hätte die glaubhafte Ablehnung eines einzelnen Mitgliedslandes wie Dänemark ausgereicht, um „Monti II“ zu kippen. Man wäre ohne Subsidiaritätskontrolle also genau zu demselben Ergebnis gelangt wie mit ihr.

Die letzte der in Abschnitt 1 vorgestellten Hoffnungen verlegte den Fokus von den Parlamenten auf die in ihnen vertretenen Parteien und zielte auf die Förderung der internen Kohärenz der konkurrierenden europäischen Parteienfamilien. Das neue Verfahren könne dazu führen, so die Hoffnung, dass die jeweiligen Schwesterparteien die Gleichgerichtetheit ihrer Interessen mehr als in der Vergangenheit erkennen, sich mehr und effektiver koordinieren, und zunehmend kohärente, unterscheidbare Programme zur europäischen Politik entwickeln. Auf diesem Wege könne, quasi als Kollateralnutzen, das Instrument auch einen Beitrag zur Weiterentwicklung der vor-konstitutionellen Voraussetzungen demokratischer Prozesse auf europäischer Ebene leisten. Vor dem Hintergrund des „Monti II“Falls zeigt sich indes, dass auch dieser Hoffnung eine unhinterfragte Prämisse zugrundliegt: die Annahme, es handle sich bei den in Frage kommenden Auseinan-

30 In dem Schreiben heißt es: "The Commission has not found based on this assessment that the principle of subsidiarity has been breached. " Der Brief ist hier online gestellt: http://ec.europa.ew/ $\mathrm{dgs} /$ secretariat_general/relations/relations_other/pdf/pdfletters/uk_house_of_commons_-_letter_ vp_sefcovic_on_monti_ii__-_withdrawal_procedure_com20120130.pdf (Zugriff: 08.06.2016).

31 ,[T]he Commission recognizes that its proposal is unlikely to gather the necessary political sup port within the European Parliament and Council to enable its adoption" (ebd.). 
dersetzungen zuvörderst um Konflikte zwischen Links und Rechts. Trifft dies zu und koordinieren sich die Parteien horizontal, mag der erhoffte Effekt einer Stärkung der internen Kohärenz der Parteienfamilien tatsächlich eintreten.

In Abschnitt 4 haben wir uns daher eingehend mit der Frage beschäftigt, welche der von "Monti II" tangierten Konfliktlinien bei der Entscheidung über die Abgabe von Rügen dominierte. Es zeigte sich, dass dies nicht so sehr die ParteienKonfliktlinie zwischen Links und Rechts war, sondern eine Konfliktlinie zwischen Ländergruppen mit unterschiedlich gelagerten Interessen. Die Existenz und die Persistenz solcher Konflikte sind in der politökonomisch heterogenen und mit unterschiedlichen Wohlstandsniveaus ausgestatteten EU nicht überraschend (Fioretos 2001; Höpner u. Schäfer 2012; Menz 2005). Zur Schärfung der Kohärenz der europäischen Parteienfamilien trägt ein Mehr an horizontaler Koordination in solchen Konstellationen aber nichts bei. Was in einer solchen Konstellation durch ein Mehr an horizontaler Koordination geschärft wird, ist keine Unterscheidbarkeit von Parteien, sondern von regionalen Interessen.

Auch hier verbietet es sich, vom Einzelfall auf die Gesamtheit der in der europäischen Politik ausgetragenen Konflikte zu schließen. Im Falle zahlreicher anderer Auseinandersetzungen, man denke etwa an den Konflikt um eine europäische Frauenquote, wäre die Hoffnung auf einen parteipolitischen Kohärenzeffekt berechtigter gewesen. Festzuhalten bleibt lediglich, dass der Kohärenzeffekt keinem Automatismus folgt. Es hängt vielmehr von der Struktur der ausgetragenen Konflikte ab, ob die verstärkte Koordination erwünschte Allianzen zwischen Parteien stärkt oder vielmehr unerwünschte Konfliktkonturen zwischen Ländergruppen schärft. Alles Weitere hinge dann von einer Gesamtevaluation der Auseinandersetzungen über europäische Politik ab, die prinzipiell zum Gegenstand der Subsidiaritätsrüge werden könnten. Eine solche Gesamtevaluation hätte freilich zu berücksichtigen, dass die durch die Eurokrise vertieften Verteilungskonflikte eine vorher nicht gekannte Spaltung der EU entlang der Nord-Süd-Achse bewirkt haben (siehe bspw. Auel u. Höing 2015; Hall 2014; Maatsch 2014).

Unsere Betrachtung gelangt also zu einem allenfalls moderat optimistischen Ergebnis. Nicht alle mit der Subsidiaritätskontrolle verknüpften Hoffnungen erscheinen bei genauerer Hinsicht berechtigt. Die Hoffnung auf eine Effektuierung des Subsidiaritätsschutzes muss mit einem großen Fragezeichen versehen werden, weil Verletzungen der Subsidiarität angesichts eines mit hohen Konsenshürden und zahlreichen Vetopunkten ausgestatteten politischen Systems weniger von der europäischen Gesetzgebung auszugehen drohen, als vielmehr von Formen der europäischen Integration, die von dem Instrument nicht erreicht werden können: von der "Integration durch Recht“ oder etwa von den neuen, aus Anlass der Eurokrise errichteten makroökonomischen Korrekturverfahren. Selbst eine Schär- 
fung der Subsidiaritätskontrolle im Zuge des so genannten „Brexit-Deals" (siehe die Einzelheiten in Höpner 2016b) würde an diesem Problem nichts ändern. ${ }^{32}$

Hofft man weniger auf einen verbesserten Autonomieschutz als vielmehr auf in den Abläufen angelegte Kommunikations-, Deliberations- und Kohärenzeffekte, dann erscheinen die auf die horizontale Ebene gerichteten Hoffnungen realistischer als die Hoffnungen, die auf den Austausch zwischen Kommission und nationalen Parlamenten gerichtet sind. Die Wirkungen eines Mehrs an horizontaler Koordination auf das demokratische Potenzial der EU sind indes eine offene Frage. Die weitreichendsten Hoffnungen richten sich auf eine Stärkung der vor-rechtlichen Voraussetzungen transnationaler demokratischer Prozesse. Dieser Effekt hängt aber wesentlich von der Struktur der ausgetragenen Konflikte ab. Handelt es sich bei ihnen um transnationale Konflikte zwischen Links und Rechts, dann mag das neue Instrument in der Tat die Kohärenz der europäischen Parteienfamilien fördern und so einen Beitrag zur Demokratisierung des politischen Systems der EU leisten. Handelt es sich hingegen - wie bei dem Konflikt um „Monti II“ um Konflikte zwischen Ländergruppen mit ähnlich gelagerten Interessen, leistet das Instrument keinen Beitrag zur internen Kohärenz der Parteienfamilien, sondern zur Schärfung innereuropäisch-regionaler Spaltungslinien.

\section{Nachwort vom Juni 2016}

Im Mai 2016 wurde zum dritten Mal die Schwelle zur „gelben Karte“ überschritten. 15 Parlamente aus 12 Ländern rügten den Kommissionsvorschlag für eine Überarbeitung der Entsenderichtlinie (RL 96/71/EG). Dieser Vorgang ist mit dem im vorliegenden Beitrag behandelten Konflikt eng verknüpft (vgl. die Angaben in Abschnitt 3 zur Deutung der Entsenderichtlinie im Laval-Urteil des EuGH). Es ist daher interessant, die Befunde des Aufsatzes gegen die neuen Erfahrungen zu prüfen.

Die neuen Rügen illustrieren, dass die Anwendungsbereiche der Subsidiaritätskontrolle über etwaige Verletzungen des Prinzips der enumerativen Einzelermächtigung weit hinausgehen und dass das Instrument daher als politischer Vetopunkt zu verstehen ist, nicht als quasi-rechtliches Instrument zur Ahndung von ultravires-Akten. Im Konflikt um die Entsenderichtlinie wird das besonders deutlich, denn der gerügte Vorschlag der Kommission würde die arbeitsrechtliche Regulierungskapazität der Mitgliedstaaten (in Antwort auf vorherige EuGH-Rechtspre-

32 Die Bestimmungen des „Brexit-Deals" werden aktiviert, wenn sich Großbritannien im für den 23. Juni 2016 angesetzten Referendum für den Verbleib in der EU ausspricht (der Ausgang des Referendums ist zum Zeitpunkt des Abschlusses dieses Manuskripts noch offen). Angestrebt wird in diesem Fall, dass Gesetzesvorschläge nicht weiter behandelt werden sollen, wenn die den nationalen Parlamenten zugewiesenen Stimmen ein Quorum von 55 Prozent übersteigen, „es sei denn, der Entwurf wird dahingehend geändert, dass den in den begründeten Stellungnahmen geäußerten Bedenken Rechnung getragen wird". Kommt es zur Aktivierung einer solchen Reform, dann hängt ihre Reichweite insbesondere davon ab, wem die Entscheidung der Frage zusteht, ob den Bedenken tatsächlich Rechnung getragen würde (ausführlich: Höpner 2016b). Der Wortlaut des vom 19.2.2016 datierenden „Brexit-Deals“ findet sich hier: http://data.consilium.europa.eu/doc/ document/ST-1-2016-INTT/de/pdf (Zugriff: 08.06.2016), die Bestimmungen zur Subsidiaritätsrüge finden sich in dem mit "Souveränität" überschriebenen Abschnitt C aus Anlage I. 
chung) im Falle der Umsetzung stärken, nicht schwächen - so dass das Vorliegen eines subsidiaritätswidrigen Übergriffs auf die mitgliedstaatlichen Rechtsordnungen kaum plausibel zu begründen ist. Im Beitrag haben wir auch aufgezeigt, dass die Subsidiaritätskontrolle nicht „beißt“", wenn etwaige Verletzungen des Subsidiaritätsprinzips vom EuGH statt von der europäischen Gesetzgebung ausgehen. Dieses Problem liegt bei der jüngsten "gelbe Karte" nicht vor. Sogar das Gegenteil ist der Fall, denn der Kommissionsvorschlag will EuGH-Rechtsprechung nicht kodifizieren, sondern behutsam korrigieren. Die Reaktion der Kommission steht zum Zeitpunkt der Niederschrift dieses Nachworts noch aus.

Zudem haben wir gezeigr, dass manche der mit der Subsidiaritätskontrolle verknüpften Hoffnungen unberechtigt sind, wenn das Instrument als Waffe in Konflikten eingesetzt wird, die nicht zwischen Links und Rechts, sondern zwischen Ländergruppen verlaufen. Dieser Einwand greift auch und gerade auf die jüngsten Vorgänge, denn noch wesentlich deutlicher als im Konflikt um „Monti II“ rügten nunmehr Parlamente aus Ländern mit ähnlichen politökonomischen Interessen - und zwar Legislativen aus Bulgarien, Dänemark, Estland, Lettland, Litauen, Kroatien, Polen, Portugal, Rumänien, der Slowakei, Tschechien und Ungarn. Gerügt haben mit Ausnahme Dänemarks also durchweg vergleichsweise niedrig produktive und arbeitsrechtlich schwach regulierte Länder mit Interesse an der Möglichkeit, schwächeres Arbeitsrecht im Zuge der Arbeitnehmerentsendung in die Mitte und den Norden der EU ,importieren“ zu können (also genau die "Spielarten des Kapitalismus"-Konfliktlinie, die wir in den Abschnitten 3 und 4 beschrieben haben). In solchen Fällen konturiert die Subsidiaritätsrüge eine innereuropäische Spaltungslinie, trägt aber nichts zur internen Kohärenz der europäischen Parteienfamilien und damit auch nichts zur Entwicklung der vor-konstitutionellen Voraussetzungen demokratischer Prozesse auf europäischer Ebene bei.

\section{Literatur}

Alter, Karen J. 2001. Establishing the Supremacy of European Law. Oxford/New York: Oxford University Press.

Amable, Bruno. 2003. The Diversity of Modern Capitalism. Oxford: Oxford University Press. DOI: 10.1093/019926113X.001.0001.

Armingeon, Klaus, Christian Isler, Laura Knöpfel, David Weisstanner, und Sarah Engler. 2015. Comparative Political Dataset III 1990-2013. Bern: Institute of Political Science, University of Berne.

Auel, Katrin, und Oliver Höing. 2015. National Parliaments and the Eurozone Crisis. Taking Ownership in Difficult Times. West European Politics 38: 375-395. DOI: 10.1080/ 01402382.2014 .990694$.

Auel, Katrin, Oliver Rozenberg, und Angela Tacea. 2015. To Scrutinze or Not to Scrutinze? Explaining Variation in EU-Related Activities in National Parliaments. West European Politics 38: 282-304. DOI: 10.1080/01402382.2014.990695.

Becker, Peter. 2013. Die Subsidiaritätsprüfung in Bundestag und Bundesrat - ein rechtliches oder ein politisches Instrument? Zeitschrift für Politikwissenschaft 23: 5-37. DOI: $10.5771 / 1430-6387-2013-1-5$. 
Boronska-Hryniewiecka, Karolina. 2013. Democratising the European Multi-level Polity? A (Re-)Assessment of the Early Warning System. Yearbook of Polish European Studies 16: 167-187.

Breukers, Romy. 2013. The Early Warning System: An Evaluation Study. Public Administration Master Thesis. Twente: Management and Governance Faculty, University of Twente.

Buschmann, Marco, und Birgit Daiber. 2011. Subsidiaritätsrüge und Grundsatz der begrenzten Einzelermächtigung. Die Öffentliche Verwaltung 13/2011: 504-509.

Chalmers, Damian. 2013. Democratic Self-Government in Europe. Domestic Solutions to the EU Legitimacy Crisis. Policy Network Paper. London: Policy Network.

Cooper, Ian. 2012. A 'Virtual Third Chamber' for the European Union? National Parliaments after the Treaty of Lisbon. West European Politics 35: 441-465. DOI: 10.1080 / 01402382.2012 .665735 .

Cooper, Ian. 2015. A Yellow Card for the Striker: National Parliaments and the Defeat of EU Legislation on the Right to Strike. Journal of European Public Policy 22: 14061425. DOI: $10.1080 / 13501763.2015 .1022569$.

Eichhorst, Werner. 2000. Europäische Sozialpolitik zwischen nationaler Autonomie und Marktfreiheit. Die Entsendung von Arbeitnehmern in der EU. Frankfurt a. M./New York: Campus.

Fioretos, Orfeo. 2001. The Domestic Sources of Multilateral Preferences: Varieties of Capitalism in the European Community. In Varieties of Capitalism. The Institutional Foundations of Comparative Advantage, Hrsg. Peter A. Hall und David Soskice, 213-244. Oxford/New York: Oxford University Press. DOI: 10.1093/0199247757.003.0006.

Gattermann, Katjana, und Claudia Hefftler. 2015. Beyond Institutional Capacity: Political Motivation and Parliamentary Behaviour in the Early Warning System. West European Politics 38: 305-334. DOI: 10.1080/01402382.2014.990696.

Goldoni, Marco. 2014. The Early Warning System and the Monti II Regulation: The Case for a Political Interpretation. European Constitutional Law Review 10: 90-108. DOI: $10.1017 / 51574019614001059$.

Groen, Afke, und Thomas Christiansen. 2015. National Parliaments in the European Union: Conceptual Choices in the European Union's Constitutional Debate. In The Palgrave Handbook of National Parliaments and the European Union, Hrsg. von Claudia Hefftler, Christine Neuhold, Olivier Rozenberg und Julie Smith, 43-59. Basingstoke: Palgrave.

Hall, Peter A. 2014. Varieties of Capitalism and the Euro Crisis. West European Politics 37: 1223-1243. DOI: 10.1080/01402382.2014.929352.

Hall, Peter A., und David Soskice. 2001. An Introduction to Varieties of Capitalism. In Varieties of Capitalism. Institutional Foundations of Comparative Advantage, Hrsg. von Peter A. Hall und David Soskice, 1-68. Oxford/New York: Oxford University Press.

Hix, Simon. 2008. The EU as a New Political System. In Comparative Politics, Hrsg. Daniele Caramani, 573-601. Oxford: Oxford University Press.

Hooghe, Liesbet, und Gary Marks. 1999. The Making of a Polity: The Struggle over European Integration. In Continuity and Change in Contemporary Capitalism, Hrsg. Herbert Kitschelt, Peter Lange, Gary Marks und John D. Stephens, 70-97. Cambridge: Cambridge University Press. 
Högenauer, Anna-Lena, und Christine Neuhold. 2015. National Parliaments after Lisbon: Administrations on the Rise? West European Politics 38: 335-354. DOI: 10.1080/ 01402382.2014.990698.

Höpner, Martin. 2016a. Das Soziale Fortschrittsprotokoll des Europäischen Gewerkschaftsbundes: Ein Vorschlag zur Weiterentwicklung. WSI-Mitteilungen 69: 245-253.

Höpner, Martin. 2016b. Reform der Europäischen Union: Rote Karte für den falschen Spieler. Makroskop vom 23.2.2016.

Höpner, Martin, und Armin Schäfer. 2012. Embeddedness and Regional Integration: Waiting for Polanyi in a Hayekian Setting. International Organization 66: 429-455. DOI: $10.1017 /$ S002081831200015X.

Höreth, Marcus. 2008. Die Selbstautorisierung des Agenten. Der Europäische Gerichtshof im Vergleich zum U.S. Supreme Court. Baden-Baden: Nomos. DOI: 10.5771/ 9783845209623.

Höscheidt, Sven. 2008. Formale Aufwcrtung - geringe Schubkraft: Die Rolle der nationalen Parlamente gemäß dem Lissabonner Vertrag. Integration 31: 254-265. DOI: 10.5771/0720-5120-2008-3-254.

Joerges, Christian, und Florian Rödl. 2009. Informal Politics, Formalised Law and the 'Social Deficit' of European Integration: Reflections after the Judgments of the ECJ in Viking and Laval. European Law Journal 15: 1-19.

Kiiver, Philipp. 2012. The Conduct of Subsidiarity Checks of EU Legislative Proposals by National Parliaments: Analysis, Observations and Practical Recommendations. ERA Forum 12: 535-547. DOI: 10.1007/s12027-011-0234-8.

Koch, Jessica, und Matthias Kullas. 2010. Subsidiarität nach Lissabon - Scharfes Schwert oder stumpfe Klinge? CEP-Studie. Freiburg: Centrum für Europäische Politik.

Leitfeld, Philip, und Thomas Malang. 2014. National Parliamentary Coordination after Lisbon: A Network Approach. Paper prepared for the $1^{\text {st }}$ European Conference on Social Networks (EUSN), 01.-04.06.2014, Barcelona.

Lijphart, Arend. 2012. Patterns of Democracy. Government Form and Performance in Thirty-Six Countries. Second Edition. New Haven: Yale University Press.

Maatsch, Aleksandra. 2014. Are We All Austerians Now? An Analysis of National Parliamentary Parties' Positioning on Anti-crisis Measures in the Eurozone. Journal of European Public Policy 21: 96-115. DOI: 10.1080/13501763.2013.829582.

Martinsen, Dorte S., und Paula Blomqvist. 2009. The European Union: Single Market Pressures. In Nordic Health Care Systems. Recent Reforms and Current Policy Challenges, Hrsg. Jon Magnussen, Karsten Vrangbæk, und Richard B. Saltman, 294-316. Maidenhead, Berkshire: Open University Press.

Menz, Georg. 2005. Varieties of Capitalism and Europeanization. National Response Strategies to the Single European Market. Oxford/New York: Oxford University Press.

Monti, Mario. 2010. Eine neue Strategie für den Binnenmarkt. Bericht an den Präsidenten der Europäischen Kommission, 9. Mai 2010, Brüssel: Europäische Kommission.

Nölke, Andreas, und Arjan Vliegenthart. 2009. Enlarging the Varieties of Capitalism. The Emergence of Dependent Market Economies in East Central Europe. World Politics 62: 670-702. DOI: 10.1017/S0043887109990098.

Pabel, Katharina. 2011. Die Kontrolle des Subsidiaritätsprinzips durch die Parlamente der Mitgliedstaaten. Journal für Rechtspolitik 19: 287-300. DOI: 10.1007/s00730-0110034-z. 
Reiding, Hilde, Wim van Meurs, und Zoe Hulsenboom. 2016. Die europäische Subsidiaritätsprüfung auf dem Prüfstand. Erwartungen und erste Erfahrungen aus dem deutschen und dem niederländischen Parlament. Zeitschrift für Parlamentsfragen 47: 85-101. DOI: 10.5771/0340-1758-2016-1-85.

Richter, Emanuel. 1987. Subsidiarität und Neokonservatismus. Die Trennung von politischer Herrschaftsbegründung und gesellschaftlichem Stufenbau. Politische Vierteljabresschrift 28: 293-314.

Rödl, Florian. 2009. Arbeitsverfassung. In Europäisches Verfassungstecht. Theoretische und dogmatische Grundzüge. 2., vollständig aktualisierte und erweiterte Auflage, Hrsg. Armin von Bogdandy und Jürgen Bast, 855-904. Berlin/Heidelberg: Springer. DOI: 10.1007/978-3-540-73810-7_18.

Sack, Detlef. 2010. Europäisierungsdruck und Parteiendifferenz in den deutschen Bundesländern. Die Rechtsprechung des EuGH und die Novellierung von Tariftreueregelungen. Politische Vierteljabresschrift 51: 619-642. DOI: 10.1007/s11615-010-0032-x.

Scharpf, Fritz W. 1985. Die Politikverflechtungs-Falle: Europäische Integration und deutscher Föderalismus im Vergleich. Politische Vierteljahresschrift 26: 323-356.

Scharpf, Fritz W.1999. Regieren in Europa: Effektiv und demokratisch? Frankfurt/New York: Campus.

Scharpf, Fritz W. 2009. Legitimität im europäischen Mehrebenensystem. Leviathan 37: 244-280. DOI: 10.1007/s11578-009-0016-7.

Schmidt, Susanne K. 2009. When Efficiency Results in Redistribution: The Conflict over the Single Services Market. West European Politics 32: 847-865. DOI: 10.1080/ 01402380902945532.

Schmidt, Susanne K. 2012. Who Cares about Nationality? The Path-dependent Case Law of the ECJ from Goods to Citizens. Journal of European Public Policy 19: 8-24. DOI: 10.1080/13501763.2012.632122.

Schreinermacher, Björn. 2014. Vom EuGH-Urteil zur Richtlinie: Wie die EU-Mitgliedstaaten über die Kodifizierung europäischer Rechtsprechung entscheiden. TranState Working Papers 184. Bremen: Universität Bremen, Sfb 597 „Staatlichkeit im Wandel“.

Seikel, Daniel, und Nadine Absenger. 2015. Die Auswirkungen der EuGH-Rechtsprechung auf das Tarifvertragssystem in Deutschland. Industrielle Beziehungen 22: 51-71. DOI: 10.1688/indb-2015-01-seikel.

Semmler, Jörg. 2010. Die Subsidiaritätsrüge nach dem Vertrag von Lissabon - Plädoyer für ein politisches Instrument. Zeitschrift für europarechtliche Studien 13: 529-538.

Shirvani, Foroud. 2010. Die curopäische Subsidiaritätsklage und ihre Umsetzung ins deutsche Recht. Juristenzeitung 65: 753-759.

Tsebelis, George, und Geoffrey Garrett. 2001. The Institutional Foundations of Intergovernmentalism and Supranationalism in the European Union. International Organization 55: 357-390. DOI: 10.1162/00208180151140603.

Visser, Jelle. 2015. ICTWSS Data Base. Version 5.0. Amsterdam: Amsterdam Institute for Advanced Labour Studies.

Weiler, Joseph H.H. 1987. The Court of Justice on Trial. On Law and Policy in the European Court of Justice. Common Market Law Review 24: 555-589. 


\section{Autorenangaben}

apl. Prof. Dr. Martin Höpner

Max-Planck-Institut für Gesellschaftsforschung

Paulstraße 3

50676 Köln

E-Mail: hoepner@mpifg.de

\section{Lena Ehret}

Freiherr-vom-Stein-Str. 21

69207 Sandhausen 
Tabelle A1: Korrelationsmatrix

\begin{tabular}{|c|c|c|c|c|c|c|c|c|c|}
\hline & $\begin{array}{c}\text { Vertrauen } \\
\text { in EU- } \\
\text { Institutio- } \\
\text { nea }\end{array}$ & $\begin{array}{c}\text { Demo- } \\
\text { kratiezu- } \\
\text { friedenheit }\end{array}$ & $\begin{array}{l}\text { Zukunfts- } \\
\text { aussichten }\end{array}$ & $\begin{array}{l}\text { Lander- } \\
\text { große }\end{array}$ & $\begin{array}{c}\text { Parteipol. } \\
\text { Zusam- } \\
\text { menset- } \\
\text { zung der } \\
\text { Regicrung }\end{array}$ & $\begin{array}{l}\text { Parlamen- } \\
\text { tarismus } \\
\text { vs. Prasi- } \\
\text { dentialis- } \\
\text { mus }\end{array}$ & $\begin{array}{l}\text { BIP pro } \\
\text { Kopf }\end{array}$ & $\begin{array}{c}\text { Gewerk. } \\
\text { Org.-Quote }\end{array}$ & $\begin{array}{c}\text { Tarif- } \\
\text { Verträge }\end{array}$ \\
\hline Vertrauen in EU-Institutionen & 1,00 & & & & & & & & \\
\hline $\begin{array}{l}\text { Zufriedenheit mit europ. } \\
\text { Demokratie }\end{array}$ & 0,43 & 1,00 & & & & & & & \\
\hline $\begin{array}{l}\text { Zukunftsaussichten } \\
\text { EU-Mitgliedschaft }\end{array}$ & 0,73 & 0,38 & 1,00 & & & & & & \\
\hline Ländergröße (Einwohner) & $-0,46$ & $-0,19$ & $-0,20$ & 1,00 & & & & & \\
\hline $\begin{array}{l}\text { Parteipol. Zusammensetzung } \\
\text { Regierung (Schmidt-Index) }\end{array}$ & 0,16 & 0,18 & 0,07 & $-0,37$ & 1,00 & & & & \\
\hline $\begin{array}{l}\text { Parlamentarismus vs. } \\
\text { Präsidentialismus }\end{array}$ & $-0,04$ & $-0,09$ & $-0,02$ & $-0,02$ & 0,33 & 1,00 & & & \\
\hline BIP pro Kopf & 0,12 & 0,50 & 0,29 & 0,04 & 0,19 & $-0,13$ & 1,00 & & \\
\hline $\begin{array}{l}\text { Gewerkschaftliche } \\
\text { Organisationsquote }\end{array}$ & 0,16 & 0,18 & 0,23 & $-0,24$ & 0,36 & $-0,04$ & 0,49 & 1,00 & \\
\hline Tarifvertragliche Deckungsrate & 0,01 & 0,33 & $-0,13$ & 0,01 & 0,22 & 0,07 & 0,34 & 0,49 & 1,00 \\
\hline
\end{tabular}

Anmerkungen: Fettóruck bei r > 0,3. Definitionen und Quellen der Variablen: siehe Angaben im Text.

Quelle: Eigene Darstellung. 